\title{
Beta wave of sleep electroencephalogram analysis based on sign series entropy
}

\author{
Min Zhao ${ }^{1}$, Yuting $\mathrm{Li}^{1}$, Lu Yang ${ }^{1}$, Jia-Fei Dai ${ }^{2, \mathrm{a}}$, Jun Wang ${ }^{1, \mathrm{~b}}$ and Feng-Zhen \\ $\mathrm{Hou}^{3, \mathrm{C}}$
}

${ }^{1}$ Image Processing and Image Communications Key Lab. , Nanjing Univ. of Posts \& Telecomm. , Nanjing 210003, China

${ }^{2}$ Nanjing General Hospital of Nanjing Military Command, Nanjing, 210002, China

${ }^{3}$ School of Science, China Pharmaceutical University, Nanjing 210009, China

a'E-mail: 37045613@qq.com, 'bangj@njupt.edu.cn, ㄷ-mail: houfz@cpu.edu.cn

Keywords: sleep EEG, sign series entropy

\begin{abstract}
Sleep and wake EEG have some differences. After studying their brain waves and calculating the sign series entropy, we use the $T$ test for the detection of sleep and wake EEG data to figure out whether they are different. After beta waves being filtered out by the filter, we calculate the entropy of the sign series. The results of $T$ test show that the beta waves in the state of sleep and wake are different.
\end{abstract}

\section{Introduction}

When a person is awake, the frequency of the brain is basically in the state of beta waves [1-4]. With the enhancement of the beta waves, the body gradually gets more nervous. In the body, the proper beta waves have a positive effect on the development of attention and cognitive behavior. When people sleep, beta waves weakened, people gradually show the state of relaxation. Through the study of the beta EEG analysis, we can distinguish that people are awake or asleep [1-4].

\section{Method of sign series entropy}

The calculation of sign series entropy [5] is first to carry out symbolic sequence. In order to represent the potential changes of the original EEG signals, its symbolic process is :

$$
r(j)= \begin{cases}0, & z(j+1)<z(j), \\ 1, & z(j+1)=z(j), \quad j=1,2,3 \ldots\left(\mathrm{L}_{m}-1\right) \\ 2, & z(j+1)>z(j),\end{cases}
$$

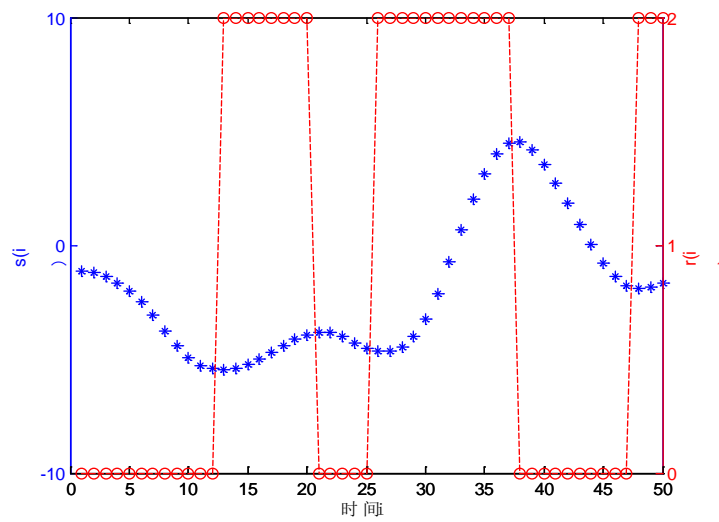

Figure 1: symbolic process of EEG signals

$R(J)=0 、 1 、 2$ represent the electric potential. The three numbers in the sign series do not have a numerical value, but are the symbols of the three directional information. In order to show the regularity of the sign series,we construct vector sequence with the width d: 


$$
R(j)=[r(j), r(j+1), \ldots, r(j+(d-1))], \quad j=1,2,3 \ldots \mathrm{L}_{m}-d
$$

When the width is $d$, the models of continuous change are $3^{d}$.For example, when $d$ is 2 , the models are $00 、 01 、 02 、 10 、 11 、 12 、 20 、 21$ and22. $D_{k}$ indicates the number of times that the models appear and the probability of each model is $\mathrm{Pk}$, which are shown in the following formula and figure:

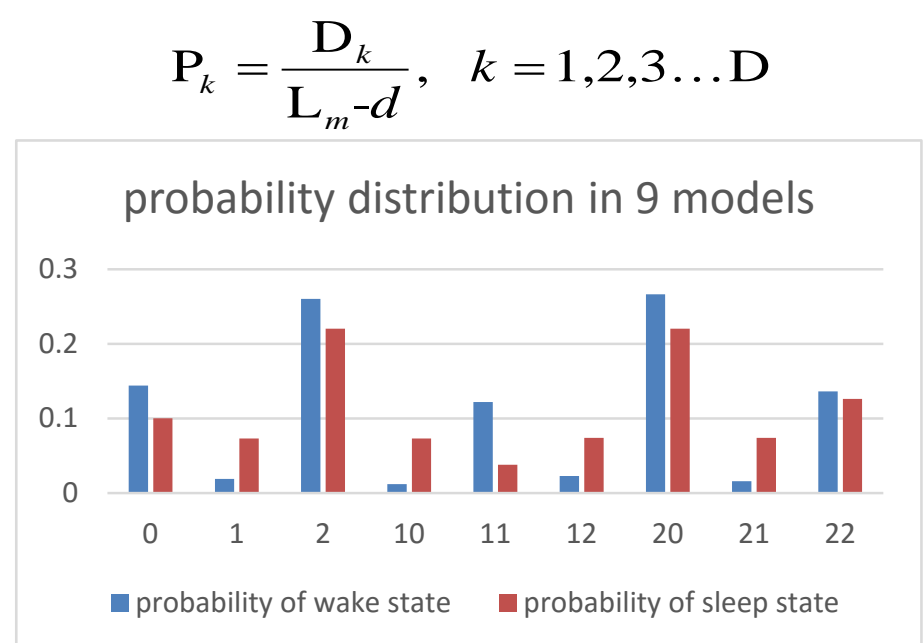

Figure 2: probability distribution of 9 models

According to the probability of each model, we can calculate the entropy:

$$
\operatorname{MSSE}(d)=-\sum_{k=1}^{\mathrm{D}}\left(\mathrm{P}_{k} \cdot \log _{2} \mathrm{P}_{k}\right)
$$

\section{Numerical analysis}

We divide collected EEG signals into 8 groups of sleep and 8 groups of wake. The width is 2 . We apply sign series entropy to the original EEG signals data for research.

Table 1: $\quad$ SSE of the data sample

\begin{tabular}{|l|c|l|l|l|l|l|l|l|l|}
\hline \multicolumn{2}{|c|}{ SSE of the data sample } & Data41 & Data411 & Data45 & Data451 & Data48 & Data481 & Data59 & Data591 \\
\hline Wake & $1 \sim 2000$ & 2.586 & 2.872 & 2.610 & 2.521 & 2.176 & 2.743 & 2.784 & 2.647 \\
\hline Sleep & $1 \sim 2000$ & 2.960 & 2.911 & 2.707 & 2.768 & 3.113 & 2.831 & 2.932 & 2.413 \\
\hline Wake & $2000 \sim 4000$ & 2.535 & 2.931 & 2.630 & 2.460 & 2.146 & 2.178 & 2.747 & 2.699 \\
\hline Sleep & $2000 \sim 4000$ & 2.824 & 2.857 & 2.804 & 2.820 & 3.087 & 2.770 & 2.925 & 2.701 \\
\hline
\end{tabular}

According to independent sample T test, we can draw the obvious difference between the sleep entropy and wake entropy. Then we divid the 8 groups of sleep and 8 groups of wake EEG data into 3 data segments whose length is 2000. After filtering out the beta component of each data segment by the filter ,we return it to the time domain.We apply sign series entropy to the beta component again. Results are shown in the following table: 
Table 2: sign series entropy of the beta wave

\begin{tabular}{|l|l|l|l|l|l|}
\hline $1 \sim 2000$ & \multicolumn{1}{|c|}{ Beta wave } & \multicolumn{1}{|c|}{ 2001 4000 } & \multicolumn{1}{|c|}{ Beta wave } & 4001 6000 & Beta wave \\
\hline 41 & 1.2783 & 41 & 1.2868 & 41 & 1.2764 \\
\hline 45 & 1.2781 & 45 & 1.2823 & 45 & 1.2606 \\
\hline 48 & 1.2588 & 48 & 1.2695 & 48 & 1.2868 \\
\hline 59 & 1.2693 & 59 & 1.2770 & 59 & 1.2731 \\
\hline 411 & 1.3034 & 411 & 1.2950 & 411 & 1.2946 \\
\hline 451 & 1.2908 & 451 & 1.2605 & 451 & 1.2867 \\
\hline 481 & 1.2949 & 481 & 1.2939 & 481 & 1.3118 \\
\hline 591 & 1.2953 & 591 & 1.2944 & 591 & 1.2952 \\
\hline $1 \sim 2000$ & Beta wave & $2001 \sim 4000$ & Beta wave & $4001 \sim 6000$ & Beta wave \\
\hline 410 & 1.2865 & 410 & & & 1.2868 \\
\hline 450 & 1.2587 & 450 & 1.2782 & 410 & 1.2739 \\
\hline 480 & 1.2866 & 480 & 1.2865 & 480 & 1.2694 \\
\hline 590 & 1.2607 & 590 & 1.2867 & 590 & 1.2780 \\
\hline 4110 & 1.2952 & 4110 & 1.2692 & 4110 & 1.2780 \\
\hline 4510 & 1.2736 & 4510 & 1.2852 & 4510 & 1.2781 \\
\hline 4810 & 1.2649 & 4810 & 1.2472 & 4810 & 1.2950 \\
\hline 5910 & 1.2782 & 5910 & 1.2605 & 5910 & 1.2604 \\
\hline
\end{tabular}

By using the independent samples $\mathrm{T}$ test for the data in the table above, we can conclude that there is a certain difference between the sleep beta component and wake beta component.

Table 3: independent samples T test for beta waves

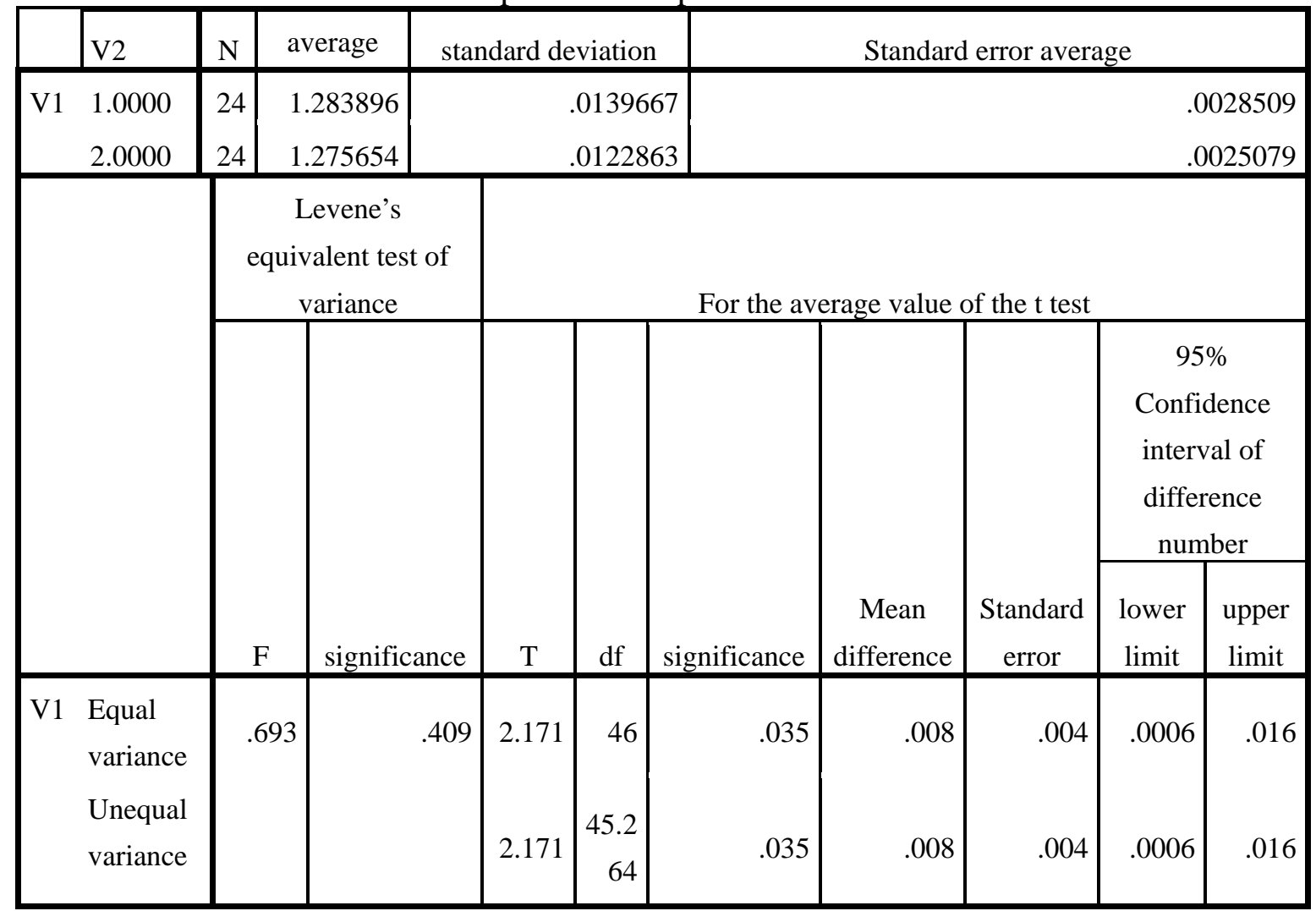

Therefore, we can get the result that beta waves based on sign series entropy can effectively distinguish sleep and wake state . 


\section{Conclusions}

In this paper, we propose sign series entropy and apply it to sleep and wake EEG analysis.The studies of this paper are based on each group whose length is 2000.We can calculate SSE values from 8 groups of sleep and 8 groups of wake EEG data.The SSE values are analyzed and the independent sample $\mathrm{T}$ test is carried out by filtering. The result of the studies shows that the values of SSE are different and it can effectively distinguish the EEG signals of beta waves in the state of sleep and wake.

\section{Acknowledgements}

Project supported by the National Natural Science Foundation of China (Grant Nos. 61271082, 61401518), Jiangsu Provincial Key R\&D Program (Social Development) (Grant No. BE2015700), the Natural Science Foundation of Jiangsu Province (Grant No. BK20141432), the Foundation of Nanjing General Hospital of Nanjing Military Command (Grant No. 2014019), Nanjing Medical Science and Technology Project (Grant No. 201503009) and the Fundamental Research Funds for the Central Universities (Grant No. FY2014LX0039).

\section{References}

[1] Siegel, J. M., Clues to the functions of mammalian sleep, Nature, 437(7063),pp 1264-1271, 2005.

[2] Borbely, A. A. \& Achermann, P., Sleep homeostasis and models of sleep regulation, J Biol. Rhythm., 14(6),pp 557-568, 1999.

[3] Czeisler, C. A., Duffy, J. F., Shanahan, T. L., Brown, E. N., Mitchell, J. F., Rimmer, D. W., Ronda, J. M., Silva, E. J., Allan, J. S., Emens, J. S., Dijk, D. J. \& Kronauer, R. E., Stability, precision, and near-24-hour period of the human circadian pacemaker. Science, 284(5423),pp. 2177-2181, 1999.

[4] Lo, C. C., Amaral, L. A. N., Havlin, S., Ivanov, P. C., Penzel, T., Peter, J. H. \& Stanley, H. E., Dynamics of sleep-wake transitions during sleep, Europhys. Lett., 57(5),pp 625-631, 2002.

[5] Bian, C., Ma, Q., Si, J., Wu, X., Shao, J., Ning, X. \& Wang, D., Sign series entropy analysis of short-term heart rate variability, Chin. Sci. Bull., 54(24),pp 4610-4615, 2009. 